\title{
Rotation sets for orbits of degree one circle maps
}

\author{
by
}

\author{
Lluís Alsedà, Moira Chas and Francesc Mañosas
}

Departament de Matemàtiques, Universitat Autònoma de Barcelona, 08193 - Bellaterra, Barcelona,

Spain

\begin{abstract}
Let $F$ be the lifting of a circle map of degree one. In [3] a notion of $F$-rotation interval of a point $x \in \mathbb{S}^{1}$ was given. In this paper we define and study a new notion of a rotation set of a point which preserves more of the dynamical information contained in the sequences $\left\{F^{n}(y)\right\}_{n=0}^{\infty}$ than the preserved by the one from [3]. In particular, we characterize dynamically the endpoints of these sets and we obtain an analogous version of the Main Theorem of [3] in our settings.
\end{abstract}

\section{Introduction and statement of the main results}

Let $\mathcal{C}_{1}\left(\mathbb{S}^{1}\right)$ be the class of all continuous maps of the circle into itself of degree one. Let $f \in \mathcal{C}_{1}\left(\mathbb{S}^{1}\right)$ and let $F: \mathbb{R} \rightarrow \mathbb{R}$ be a lifting of $f$. Denote by $\pi$ the canonical projection from $\mathbb{R}$ to $\mathbb{S}^{1}$. It is well known that, when $f$ is a homeomorphism, then $\lim _{n \rightarrow \infty} \frac{F^{n}(y)-y}{n}$ exists, it does not depend on $y$ and is called the rotation number of $f$. In the general case of endomorphisms, this limit may not exist. Newhouse, Palis and Takens introduced in [10] the notion of rotation set of an endomorphism, $L_{F}$, by defining:

$$
L_{F}=\mathrm{Cl}\left(\left\{\rho_{F}^{+}(x): x \in \mathbb{S}^{1}\right\}\right)
$$

where $\mathrm{Cl}(\cdot)$ means topological closure and

$$
\rho_{F}^{+}(x)=\limsup _{n \rightarrow \infty} \frac{F^{n}(y)-y}{n}
$$

for any $y \in \pi^{-1}(x)$ (note that since $f$ has degree one, $F(y+1)=F(y)+1$ for each $y \in \mathbb{R}$ and, hence, $\rho_{F}^{+}(x)$ does not depend on $y$ ). They also proved that $L_{F}$ is an interval. Clearly, $L_{F}$ is defined up to translations by integers. In [6] Ito proved that 
each $\alpha \in L_{F}$ is realized as the rotation number of some point in $\mathbb{S}^{1}$ in the sense that, for some $x \in \mathbb{S}^{1}, \alpha=\lim _{n \rightarrow \infty} \frac{F^{n}(y)-y}{n}$, where $y \in \pi^{-1}(x)$. In such a case we will say that $\alpha$ is the rotation number of $x$ and we will denote it by $\rho_{F}(x)$. We note that each point in the orbit of $x$ has the same rotation number. So, we often will talk about the rotation number of an orbit and will use the notation $\rho_{F}(P)$ with $P$ the orbit of a point $x \in \mathbb{S}^{1}$.

Bamon, Malta, Pacífico and Takens defined in [3] the notion of rotation set of a point $x \in \mathbb{S}^{1}$, which will be denoted here by $L_{F}(x)$, as the set of limit points of the sequence $\left\{\frac{F^{n}(y)-y}{n}\right\}_{n=0}^{\infty}$, where $y \in \pi^{-1}(x)$. They prove that it is a closed subinterval of $L_{F}$ and that given $[\alpha, \beta] \subset L_{F}, \alpha \leq \beta$, there exists $x \in \mathbb{S}^{1}$ such that $L_{F}(x)=[\alpha, \beta]$.

We are interested in obtaining more dynamical information from the sequence $\left\{F^{n}(y)\right\}_{n=0}^{\infty}$. More precisely, we are looking for a larger set of rotation numbers which could be calculated from such a sequence (and, of course, included in $L_{F}$ ). We do it by considering not only the rotation numbers of the $f$-orbit of $x$ but the rotation numbers of the closure of such orbit. This last set of rotation numbers can be easily calculated using only the numerical values of the sequence $\left\{F^{n}(y)\right\}_{n=0}^{\infty}$ in the following way: Given a set $C \in \mathbb{S}^{1}$, consider the limits of all convergent sequences of the form:

$$
\frac{F^{n_{i}+m_{i}}\left(y_{i}\right)-F^{m_{i}}\left(y_{i}\right)}{n_{i}} \text {, with } n_{i} \rightarrow \infty, \pi\left(y_{i}\right) \in C \text { and } m_{i} \in \mathbb{N} \cup\{0\} \text {. }
$$

The set obtained in such a way will be called the rotation set of $C$ and will be denoted by $R_{F}(C)$. To simplify the notation, in the case that $C=\{x\}$ we will simply write $R_{F}(x)$ instead of $R_{F}(\{x\})$. It is straightforward to see that $L_{F}(x) \subset R_{F}(x)$ but the inclusion may be strict as it is shown in the example we exhibit in Section 4.

Another way to express our definition is the following:

$$
R_{F}(C)=\bigcap_{n=0}^{\infty} \overline{\bigcup_{m \geq n} K_{m}(C)} \text { where } K_{m}(C)=\left\{\frac{F^{m}(y)-y}{m}: \pi(y) \in \cup_{n=0}^{\infty} f^{n}(C)\right\} \text {. }
$$

From this definition it follows immediately that the set $R_{F}(C)$ is closed.

Both forms of the definition of $R_{F}(C)$ are similar to the one given in [8] for rotation sets of $k$-dimensional torus endomorphisms. Nevertheless, their definition is "global" (they take $C=\mathbb{T}^{k}$ ) and we are going to work in a "local" context because our principal interest is to study the case $C=\{x\}$. In the global context studied in [8] it is proved that $R_{F}\left(\mathbb{S}^{1}\right)=L_{F}$. A consequence of this equality is that for any $C \subset \mathbb{S}^{1}, R_{F}(C) \subset L_{F}$.

The aim of this paper is to study the set defined above. In particular, we characterize dynamically the endpoints of the sets of the form $R_{F}(x)$ and we obtain an analogous version of the Main Theorem of [3] in our settings. This is achieved in Theorems A and $\mathrm{B}$ below.

We recall that the $\omega$-limit of $x$, denoted by $\omega_{f}(x)$, is the set of points $y \in \mathbb{S}^{1}$ for which there exists an strictly increasing sequence of natural numbers $\left\{n_{k}\right\}_{k \in \mathbb{N}}$ such that 
$\lim _{k \rightarrow \infty} f^{n_{k}}(x)=y$. If $x \in \omega_{f}(x)$ we will say that $x$ is recurrent. By $\operatorname{Conv}(C)$ we will mean the convex hull of the set $C$.

Theorem A Let $f \in \mathcal{C}_{1}\left(\mathbb{S}^{1}\right)$ and let $x \in \mathbb{S}^{1}$. Then there exist two recurrent points $u, v \in \omega_{f}(x)$ such that

$$
R_{F}(x)=[\rho(u), \rho(v)]=\operatorname{Conv}\left(\bigcup_{z \in \omega_{f}(x)} L_{F}(z)\right) .
$$

Theorem A will follow immediately as a corollary of a more general theorem which will be stated and proved in Section 2. The example in Section 4 shows that without taking the convex hull of the set $\cup_{z \in \omega_{f}(x)} L_{F}(z)$ Theorem A is no longer valid.

Theorem B Let $f \in \mathcal{C}_{1}\left(\mathbb{S}^{1}\right)$ and let $F$ be a lifting of $f$. If $[\alpha, \beta] \subset L_{F}$ then there exists $x \in \mathbb{S}^{1}$ such that

$$
L_{F}(x)=R_{F}(x)=[\alpha, \beta]
$$

The paper is organized as follows: in Section 2 we prove the mentioned stronger version of Theorem A and in Section 3 we prove Theorem B. In each case we develop the necessary tools. Section 4 is devoted to exhibit the above mentioned example.

\section{Characterization of the set $R_{F}(C)$}

The aim of this section is to prove the next theorem from which Theorem A can be easily deduced. In what follows, the arcs of $\mathbb{S}^{1}$ will be called intervals. Also, given $C \subset \mathbb{S}^{1}$ we will denote by $W(C)$ the set $\mathrm{Cl}\left(\cup_{n=0}^{\infty} f^{n}(C)\right)$.

Theorem 2.1. Let $f \in \mathcal{C}_{1}\left(\mathbb{S}^{1}\right)$ and let $C \subset \mathbb{S}^{1}$ be an interval, possibly degenerated to a point. Then there exist recurrent points $u, v \in W(C)$ such that

$$
R_{F}(C)=R_{F}(\mathrm{Cl}(C))=[\rho(u), \rho(v)]=\operatorname{Conv}\left(\bigcup_{z \in \widetilde{\omega}(C)} L_{F}(z)\right)
$$

where $\widetilde{\omega}(C)=\cup_{x \in C} \omega_{f}(x)$.

To prove Theorem 2.1 we need some preliminary results.

Lemma 2.2. Let $F$ be a lifting of a map $f \in \mathcal{C}_{1}\left(\mathbb{S}^{1}\right)$ and let $C \subset \mathbb{S}^{1}$. Then $R_{F}(C)=$ $R_{F}(\mathrm{Cl}(C))$.

Proof. The continuity of $f$ implies that:

$$
\cup_{n=0}^{\infty} f^{n}(\mathrm{Cl}(C)) \subset \cup_{n=0}^{\infty} \mathrm{Cl}\left(f^{n}((C))\right) \subset W(C) .
$$


Hence, if we set $g(x)=\frac{F^{m}(x)-x}{m}$ then $K_{m}(C)=g\left(\cup_{n=0}^{\infty} f^{n}(C)\right)$. From the above inclusion and the continuity of $g$ we deduce that:

$$
g\left(\cup_{n=0}^{\infty} f^{n}(\mathrm{Cl}(C))\right) \subset \mathrm{Cl}\left(g\left(\cup_{n=0}^{\infty} f^{n}(C)\right)\right) .
$$

Therefore, $\cup_{m \geq n} K_{m}(\mathrm{Cl}(C)) \subset \mathrm{Cl}\left(\cup_{m \geq n} K_{m}(C)\right)$ and, hence, $R_{F}(\mathrm{Cl}(C)) \subset R_{F}(C)$. Since the other inclusion is obvious the lemma holds.

Note that, from the above lemma, we see that $R_{F}(C)=R_{F}(W(C))$ for each $C \subset \mathbb{S}^{1}$.

Given a lifting $F$ of $f \in \mathcal{C}_{1}\left(\mathbb{S}^{1}\right)$ we define the displacement function $\varphi: \mathbb{S}^{1} \longrightarrow \mathbb{R}$ as $\varphi(x)=F(y)-y$ where $y \in \pi^{-1}(x)$. This function is continuous and, as it is easy to see, verifies that $F^{n}(y)-y=\sum_{i=0}^{n-1} \varphi\left(f^{i}(\pi(y))\right)$ for each $n \in \mathbb{N}$ and $y \in \mathbb{R}$. Moreover, since $f$ has degree one it does not depend on the choice of $y$. This equality suggests, as it is remarked in [5], that the problems of convergence of the sequence $\left\{\frac{F^{n}(y)-y}{n}\right\}_{n \in \mathbb{N}}$ are deeply related with Birkhoff's Ergodic Theorem (see [12] for the precise statement).

If $C$ is a subset of $\mathbb{S}^{1}$, we denote by $M_{e}^{f}(C)$ and $M_{m}^{f}(C)$ the spaces of ergodic and invariant probabilistic measures for $\left.f\right|_{W(C)}$ respectively. It is well known that the extremal points of $M_{m}^{f}(C)$ belong to $M_{e}^{f}(C)$ and that $\operatorname{Conv}\left(M_{e}^{f}(C)\right)=M_{m}^{f}(C)$ (see [12]). In this context, we define the following sets:

$$
\begin{aligned}
& R_{F}^{e}(C)=\left\{\int_{W(C)} \varphi d \mu: \mu \in M_{e}^{f}(C)\right\}, \\
& R_{F}^{m}(C)=\left\{\int_{W(C)} \varphi d \mu: \mu \in M_{m}^{f}(C)\right\} .
\end{aligned}
$$

The next result was proved for $k$-dimensional torus homeomorphisms homotopic to the identity in the case $C=\mathbb{T}^{k}$ by Misiurewicz and Ziemian in [8]. The proof we present here follows ideas that Fathi developed in [5] for the global case. We note that Lemma 2.3 and its proof are valid not only for the circle but also in the $k$-dimensional torus.

Lemma 2.3. Let $C$ be a closed interval of $\mathbb{S}^{1}$ and let $F$ be a lifting of a map $f \in \mathcal{C}_{1}\left(\mathbb{S}^{1}\right)$. Then:

$$
R_{F}^{e}(C) \subset R_{F}(C) \subset R_{F}^{m}(C) .
$$

Moreover, $R_{F}^{m}(C)=\operatorname{Conv}\left(R_{F}^{e}(C)\right)$.

Proof. If $\mu \in M_{e}^{f}(C)$, by Birkhoff's Ergodic Theorem, for $\mu$-almost every $x \in W(C)$ we have that

$$
\frac{F^{n}(y)-y}{n}=\frac{1}{n} \sum_{i=0}^{n-1} \varphi\left(f^{i}(x)\right) \rightarrow \int_{W(C)} \varphi d \mu
$$

where $y \in \pi^{-1}(x)$. Therefore, $\int_{W(C)} \varphi d \mu \in R_{F}(W(C))=R_{F}(C)$. Hence, the first inclusion holds. 
Denote by $\delta_{x}$ the measure defined by

$$
\delta_{x}(U)= \begin{cases}1 & \text { if } x \in U \\ 0 & \text { otherwise }\end{cases}
$$

for each $U \subset W(C)$.

To prove the second inclusion, we take $x_{j} \in \cup_{n=0}^{\infty} f^{n}(C)$ and a strictly increasing sequence $\left\{n_{j}\right\}_{j=1}^{\infty} \subset \mathbb{N}$ such that $\lim _{j \rightarrow \infty} \frac{F^{n_{j}}\left(y_{j}\right)-y_{j}}{n_{j}}$ exists, where $y_{j} \in \pi^{-1}\left(x_{j}\right)$. Then observe that

$$
\frac{F^{n_{j}}\left(y_{j}\right)-y_{j}}{n_{j}}=\frac{1}{n_{j}} \sum_{i=0}^{n_{j}-1} \varphi\left(f^{i}\left(x_{j}\right)\right)=\int_{W(C)} \varphi d\left(\frac{1}{n_{j}} \sum_{i=0}^{n_{j}-1} \delta_{f^{i}\left(x_{j}\right)}\right) .
$$

Moreover, $M_{m}^{f}(C)$ is compact with the weak*-topology. So, there exist a subsequence of $\left\{\frac{1}{n_{j}} \sum_{i=0}^{n_{j}-1} \delta_{f^{i}\left(x_{j}\right)}\right\}_{j \in \mathbb{N}}$ which converges to an $f$-invariant probability measure on $W(C)$. So, the second inclusion holds.

To prove the last statement we note that the map $\mu \rightarrow \int_{W(C)} \varphi d \mu$ is linear and continuous. Hence, $R_{F}^{m}(C)$ is convex and compact because so is $M_{m}^{f}(C)$ (recall that $\left.\operatorname{Conv}\left(M_{e}^{f}(C)\right)=M_{m}^{f}(C)\right)$. Now take an extremal point $a \in R_{F}^{m}(C)$ and denote by $M_{a}$ the set of $\mu \in M_{m}^{f}$ such that $\int_{W(C)} \varphi d \mu=a$. We claim that $M_{a}$ contains an extremal point of $M_{m}^{f}$. Otherwise, because of the Krein-Millman's Theorem [11], each $\mu \in M_{a}$, is a non-trivial convex combination of finitely many extremal points $\mu_{1}, \mu_{2}, \ldots, \mu_{l}$ from $M_{m}^{f}$. Note that, for $i=1,2, \ldots, l, \mu_{i} \notin M_{a}$ because it is extremal. Therefore, since the map $\mu \rightarrow \int_{W(C)} \varphi d \mu$ is linear, we get that $a$ is a non-trivial convex combination of points in $R_{F}^{m}(C)$ all of them different from $a$. This contradicts the extremality of $a$ and the claim holds. Thus, in $M_{a}$ there exist an ergodic measure and, hence, $R_{F}^{e}$ contains the extremal points of $R_{F}^{m}$. Putting all together we get the last statement of the lemma.

It was proved in [9] that for two-dimensional torus homeomorphisms homotopic to the identity, the interiors of $R_{F}^{e}\left(\mathbb{T}^{2}\right)$ and $R_{F}^{m}\left(\mathbb{T}^{2}\right)$ coincide. This result is no longer valid in our context (see, for instance, the example in Section 4).

The proof of the next lemma follows standard ideas that can be found in [7] and [8]. We need the following notions. Assume that $A \subset \mathbb{R}$ and let $x, y \in A$. An $\varepsilon$-chain in $A$ between $x$ and $y$ will be a finite subset $\left\{x=x_{1}, \ldots, x_{m}=y\right\} \subset A$ such that $\left|x_{i}-x_{i+1}\right|<\varepsilon$ for $i=1, \ldots, m-1$. A set $A \subset \mathbb{R}$ will be called $\varepsilon$-chained if for any $x, y \in A$ there exists an $\varepsilon$-chain in $A$ between $x$ and $y$.

Lemma 2.4. Let $f \in \mathcal{C}_{1}\left(\mathbb{S}^{1}\right)$ and let $C$ be a connected subset of $\mathbb{S}^{1}$. Then, for each lifting $F$ of $f, R_{F}(C)$ is a closed interval of $\mathbb{R}$.

Proof. It is an easy exercise to prove the following assertions:

(i) If $A$ is $\varepsilon$-chained then $\mathrm{Cl}(A)$ is also $\varepsilon$-chained. 
(ii) Assume that $\left\{A_{i}\right\}_{i \in \mathbb{N}}$ is a sequence of subsets of $\mathbb{R}$, decreasing by inclusion and such that for every $\varepsilon>0$ there exist $i \in \mathbb{N}$ such that the set $A_{i}$ is $\varepsilon$-chained. Then $\cap_{i \in \mathbb{N}} A_{i}$ is $\varepsilon$-chained for each $\varepsilon>0$.

(iii) If a set $A \subset \mathbb{R}$ is $\varepsilon$-chained for every $\varepsilon>0$ then it is connected.

Therefore, we only have to prove that for every $\varepsilon>0$ there exist $n \in \mathbb{N}$ such that $\bigcup_{m>n} K_{m}(C)$ is $\varepsilon$-chained. In what follows, to simplify the notation, for $m \in \mathbb{N}$ and $x \in \mathbb{S}^{1}$ we will set $A_{m}(x)=\frac{F^{m}(y)-y}{m}$, where $y \in \pi^{-1}(x)$. As above, since $f$ has degree one, $A_{m}(x)$ does not depend on the choice of $y$. An easy calculation proves that $\left|A_{k}(x)-A_{k}(f(x))\right| \leq \frac{2|\varphi|}{k}$ and $\left|A_{k}(x)-A_{k+1}(x)\right| \leq \frac{|\varphi|}{k}$ for each $k \in \mathbb{N}$ and $x \in \mathbb{S}^{1}$. So, given $\varepsilon>0, k$ large enough and $j \in \mathbb{N}$ there exist $\varepsilon$-chains between $A_{k}(x)$ and $A_{k}\left(f^{j}(x)\right)$ and between $A_{k}(x)$ and $A_{k+j}(x)$. Besides, for each $i \in \mathbb{N}$, there is an $\varepsilon$-chain between $A_{i}(u)$ and $A_{i}(v)$ if $u$ and $v$ belong to $f^{l}(C)$ for some $l$. Consequently, if $x \in f^{i}(C)$, $y \in f^{i+j}(C), m_{1}, m_{2} \in \mathbb{N}$ and $m_{1}, m_{2}>k$ we have the following diagram (where each arrow means that there is an $\varepsilon$-chain between the elements it joins): $A_{m_{1}}(x) \rightarrow$ $A_{m_{1}}\left(f^{j}(x)\right) \rightarrow A_{m_{1}}(y) \rightarrow A_{m_{2}}(y)$. In short we have proved that $\bigcup_{m>n} K_{m}(C)$ is $\varepsilon$ chained.

In what follows, if $f$ is a map, we will denote by $\operatorname{Rec}(f)$ the set of all recurrent points of $f$.

Lemma 2.5. Let $f \in \mathcal{C}_{1}\left(\mathbb{S}^{1}\right)$ have a lifting $F$ and let $C$ be a closed subset of $\mathbb{S}^{1}$. Then, for each extremal point $\alpha$ of $R_{F}^{m}(C)$, there exist $x \in \operatorname{Rec}\left(\left.f\right|_{W(C)}\right)$ such that $\rho_{F}(x)=\alpha$.

Proof. If $a$ is an extremal point of $R_{F}^{m}(C)$ then, by Lemma 2.3, $a \in R_{F}^{e}(C)$. So, by Birkhoff's Ergodic Theorem there exist an ergodic measure $\mu$ and a set $B \subset W(C)$ of $\mu$ measure 1 such that for every $x \in B, a=\lim _{m \rightarrow \infty} \frac{1}{m} \sum_{i=0}^{m-1} \varphi\left(f^{i}(x)\right)=\int_{W(C)} \varphi d \mu$. The set $\operatorname{Rec}\left(\left.f\right|_{W}(C)\right)$ has $\mu$-measure equal to one (see Theorem 6.15(i) and the remark after it of [12]). Consequently, $B \cap \operatorname{Rec}\left(\left.f\right|_{W}(C)\right)$ is non-empty and for all $y \in B \cap \operatorname{Rec}\left(\left.f\right|_{W}(C)\right)$ we have that $\rho(y)=a$.

Proof of Theorem 2.1. By Lemmas 2.2 and 2.4 there exist $a, b \in \mathbb{R}$ such that

$$
R_{F}(\mathrm{Cl}(C))=R_{F}(C)=[a, b]
$$

Besides, by Lemma 2.3, we have that

$$
\operatorname{Conv}\left(R_{F}^{e}(\mathrm{Cl}(C))\right)=\operatorname{Conv}\left(R_{F}(\mathrm{Cl}(C))\right)=[a, b]=R_{F}^{m}(\mathrm{Cl}(C)) .
$$

This implies that $a$ and $b$ are the extremal points of $R_{F}^{m}(\mathrm{Cl}(C))$. Thus, from Lemma 2.5, there exist recurrent points $u, v \in W(C)$ such that $a=\rho(u)$ and $b=\rho(v)$. Since $\widetilde{\omega}(C) \subset W(C)$, from the definitions we get that $\bigcup_{z \in \widetilde{\omega}(C)} L_{F}(z) \subset R_{F}(W(C))=R_{F}(C)$. Therefore, $\operatorname{Conv}\left(\bigcup_{z \in \widetilde{\omega}(C)} L_{F}(z)\right) \subset R_{F}(C)$. On the other hand, since $u, v \in \widetilde{\omega}(C)$, $R_{F}(C)=\operatorname{Conv}(\rho(u), \rho(v)) \subset \operatorname{Conv}\left(\bigcup_{z \in \widetilde{\omega}(C)} L_{F}(z)\right)$. This ends the proof of the theorem. 


\section{Proof of theorem B}

To prove Theorem $\mathrm{B}$ we need to introduce again some notions.

If $I$ and $J$ are intervals of $\mathbb{S}^{1}$ and $f \in \mathcal{C}_{1}\left(\mathbb{S}^{1}\right)$ we say that $I f$-covers $J$ if there exists a subinterval $K$ of $I$ such that $f(K)=J$. We say that $I$ f-covers $J n$ times if there exist $n$ subintervals $K_{1}, \ldots, K_{n}$ of $I$ with pairwise disjoint interiors such that $f\left(K_{i}\right)=J$.

Let $P$ be a finite subset of $\mathbb{S}^{1}$. A $P$-basic interval is an interval $I$ of $\mathbb{S}^{1}$ having both endpoints in $P$ and such that it does not contain any point of $P$ in its interior. The partition determined by $P$ is defined to be the set of all $P$-basic intervals. The $P$-graph of a map $f \in \mathcal{C}_{1}\left(\mathbb{S}^{1}\right)$ is an oriented generalized (i.e., with several arrows joining the same vertices) graph with vertices $I_{1}, \ldots, I_{n}$ such that there are $n$ arrows from $I_{i}$ to $I_{j}$ if $I_{i}$ covers $I_{j} n$ times but not $n+1$ times. A path is a finite sequence of $P$-basic intervals $I_{i_{1}}, \ldots, I_{i_{n}}$ such that for every $1 \leq j<n$ there exists an arrow from $I_{i_{j}}$ to $I_{i_{j+1}}$.

Let $P \subset \mathbb{S}^{1}$ be a finite set such that $0 \in \pi^{-1}(P)$. If $J$ is a subset of a $P$-basic interval, we define $\widetilde{J}$ as $\pi^{-1}(J) \cap[0,1]$. Suppose now that $F$ is a lifting of a map $f \in \mathcal{C}_{1}\left(\mathbb{S}^{1}\right)$ and consider two $P$-basic intervals $I$ and $J$. If in the oriented generalized $P$-graph of $f$ there are exactly $m$ arrows from $I$ to $J$, take the $m$ corresponding subintervals $K_{1}, \ldots, K_{m}$ of $I$ with pairwise disjoint interiors such that $f\left(K_{i}\right)=J$ for each $i=1, \ldots, m$. There exist integer numbers $k_{1}, \ldots, k_{m}$ verifying that $F\left(\widetilde{K}_{i}\right)=\widetilde{J}+k_{i}$. We define the rotational P-graph of $f$ as the directed labeled $P$-graph of $f$ where the arrow from $I$ to $J$ corresponding to the interval $K_{i}$ is labeled with the integer $k_{i}$. We will say that a sequence of the form $A_{1}^{k_{1}} \ldots A_{n}^{k_{n}}$ where the $A_{i}^{\prime} s$ are $P$-basic intervals and the $k_{i}^{\prime} s$ are integer numbers is admissible for the rotational $P$-graph of $f$ (or, if there is no confusion, admissible for $f$ ) if for every $i=1, \ldots, n-1$ there is an arrow from $A_{i}$ to $A_{j}$ with label $k_{i}$ in the rotational $P$-graph of $f$. (Observe that an admissible sequence corresponds to a path in the rotational $P$-graph of $f$ ).

Consider a sequence $\left\{P_{i}\right\}_{i=1}^{n}$ of finite subsets of $\mathbb{S}^{1}$ such that $0 \in \pi^{-1}\left(P_{i}\right)$ for each $i=1,2, \ldots, n$. Let $\underline{\mathbf{A}}=A_{1}^{k_{1}} \ldots A_{n}^{k_{n}}$ be a sequence such that each $A_{i}$ is a $P_{i}$-basic interval and $k_{1}, k_{2}, \ldots, k_{n}$ are integer numbers. The number $n$ will be called the length of the sequence $\underline{\mathbf{A}}$ and will be denoted by $\operatorname{len}(\underline{\mathbf{A}})$. The rotation number of $\underline{\mathbf{A}}$, denoted

by $\rho(\underline{\mathbf{A}})$, is defined to be the rational number $\frac{\sum_{j=1}^{n} k_{j}}{n}$. Assume that $\underline{\mathbf{B}}=B_{1}^{m_{1}} \ldots B_{n}^{m_{n}}$ also satisfies that each $B_{i}$ is a $P_{i}$-basic interval and $m_{1}, m_{2}, \ldots, m_{n}$ are integer numbers. The concatenation $A_{1}^{k_{1}} \ldots A_{n}^{k_{n}} B_{1}^{m_{1}} \ldots B_{n}^{m_{n}}$ of $\underline{\mathbf{A}}$ and $\underline{\mathbf{B}}$ will be denoted by $\underline{\mathbf{A B}}$. We also will denote the concatenation of $\underline{\mathbf{A}}$ with itself $m \geq 1$ times as $\underline{\mathbf{A}}^{m}$. Let $F$ be a lifting of a map $f \in \mathcal{C}_{1}\left(\mathbb{S}^{1}\right)$. We say that a point $x \in \mathbb{S}^{1} f$-follows the sequence $\underline{\mathbf{A}}$ if for every $1 \leq i \leq n$ and $y \in \pi^{-1}(x) \cap[0,1]$ we have that $F^{i-1}(y) \in \widetilde{A}_{i}+\sum_{j=1}^{i-1} k_{j}$.

Lemma 3.1. Let $\left\{k_{i}\right\}_{i \in \mathbb{N}} \subset \mathbb{N}$ and let $A_{1}, A_{2}, \ldots$ be an infinite sequence such that, for each $i \in \mathbb{N}, A_{i}$ is a $P_{i}$-basic interval for some finite subset $P_{i} \subset \mathbb{S}^{1}$ such that $0 \in \pi^{-1}\left(P_{i}\right)$. Let $f \in \mathcal{C}_{1}\left(\mathbb{S}^{1}\right)$ and assume that a point $x \in \mathbb{S}^{1} f$-follows the sequence 
$A_{1}^{k_{1}} A_{2}^{k_{2}} \ldots A_{n}^{k_{n}}$ for each $n \in \mathbb{N}$. Then, for each lifting $F$ of $f$, the rotation set of $x$, $L_{F}(x)$, is the set of accumulation points of the sequence $\left\{\frac{\sum_{i=1}^{n} k_{i}}{n}\right\}_{n \in \mathbb{N}}$ and $R_{F}(x)$ is the set of accumulation points of all sequences of the form $\left\{\frac{\sum_{i=m_{i}}^{n_{i}+m_{i}} k_{i}}{n_{i}}\right\}_{i \in \mathbb{N}}$, where $n_{i} \rightarrow \infty$ as $i \rightarrow \infty$ and $m_{i} \in \mathbb{N}$.

Proof. Since $f$ has degree one we have that

(i) for every $i \in \mathbb{N},\left|k_{i}\right| \leq \max _{x \in[0,1]}|F(x)|+1$, and

(ii) for every $n \in \mathbb{N},\left|F^{n}(x)-\sum_{i=1}^{n} k_{i}\right| \leq 1$.

So the statement can be easily deduced from the these observations.

A point $x$ is periodic of period $n$ if $f^{n}(x)=x$ and $f^{i}(x) \neq x$ if $1 \leq i<n$. The orbit of a point $x \in \mathbb{S}^{1}$ will be denoted as $\operatorname{Orb}_{f}(x)$. The orbit of a periodic point will be called a periodic orbit.

Assume that $P$ is a periodic orbit of a map $f \in \mathcal{C}_{1}\left(\mathbb{S}^{1}\right)$ with rotation number $\frac{p}{q}$ and that $P \subset Q$ for some finite subset $Q$ of $\mathbb{S}^{1}$. We note that for each $x \in P$ there exists an admissible sequence $\underline{\mathbf{A}}=A_{1}^{k_{1}} A_{2}^{k_{2}} \ldots A_{n}^{k_{n}}$ for the rotational $Q$-graph of $f$ such that $\rho(\underline{\mathbf{A}})=\frac{p}{q}$ and the point $x f$-follows the sequence $\underline{\mathbf{A}}^{m}$ for every $m \in \mathbb{N}$. Such a sequence $\underline{\mathbf{A}}$ will be called the code of $x$ in the rotational $Q$-graph of $f$.

The proof of the next lemma follows easily from Lemma 1.2.6 of [1].

Lemma 3.2. If $\underline{\mathbf{A}}=A_{1}^{k_{1}} \ldots A_{n}^{k_{n}}$ is an admissible sequence for the rotational $P$-graph of $f$ then there exists $I \subset A_{1}$ such that every $x \in I f$-follows $\underline{\mathbf{A}}$ and $F^{n-1}(\widetilde{I})=$ $\widetilde{A}_{n}+\sum_{i=1}^{n-1} k_{i}$.

Let $f \in \mathcal{C}_{1}\left(\mathbb{S}^{1}\right)$ and let $F$ be a lifting of $f$. We say that the orbit of a point $x \in \mathbb{S}^{1}$ is twist if $\left.F\right|_{\pi^{-1}\left(\operatorname{Orb}_{f}(x)\right)}$ is non-decreasing. A periodic orbit $P$ of $f$ of period $n$ is said to be irreducible if there does not exist $m<n, x \in \mathbb{R}$ and a set of closed disjoint intervals $\left\{I_{1}, I_{2}, \ldots, I_{m}\right\} \subset[x, x+1]$ such that for each $i \in\{1, \ldots, m\}$ there exist $j \in\{1, \ldots, m\}$ and $k \in \mathbb{Z}$ verifying that $F\left(\pi^{-1}(P) \cap I_{i}\right)=\left(\pi^{-1}(P) \cap I_{j}\right)+k$.

If $f, g \in \mathcal{C}_{1}\left(\mathbb{S}^{1}\right)$ are two degree one circle maps and $P \subset \mathbb{S}^{1}$, we will say that the rotational $P$-graph of $g$ is a subgraph of the rotational $P$-graph of $f$ if for each labeled arrow of the rotational $P$-graph of $g$ there is an arrow of the rotational $P$-graph of $f$ joining the same vertices with the same label. A graph is strongly connected if there is a finite path between each pair of vertices. The following lemma studies some conditions to assure that a graph has this property.

Lemma 3.3. Assume that $P_{1}, \ldots, P_{n}$ are irreducible periodic orbits of a map $f \in \mathcal{C}_{1}\left(\mathbb{S}^{1}\right)$ with different rotation numbers. Then the $\cup_{k=1}^{n} P_{k}$-graph of $f$ is strongly connected

Proof. Let $F$ be a lifting of $f$ and consider the continuous map $G: \mathbb{R} \rightarrow \mathbb{R}$ defined as follows: $G(x)=F(x)$ if $x \in \pi^{-1}\left(\cup_{k=1}^{n} P_{k}\right)$ and $G$ is affine on each connected component 
of $\mathbb{R} \backslash \pi^{-1}\left(\cup_{k=1}^{n} P_{k}\right)$. The map $G$ turns to be the lifting of a map $g \in \mathcal{C}_{1}\left(\mathbb{S}^{1}\right)$ and its $\cup_{k=1}^{n} P_{k}$-graph is a subgraph of the $\cup_{k=1}^{n} P_{k}$-graph of $f$. Then if we prove that the $\cup_{i=1}^{n} P_{i}$-graph of $g$ is strongly connected we are done. Suppose that it is not strongly connected. Then there are two basic intervals $I$ and $J$ such that there is no path from $I$ to $J$. Let $W=\cup_{i=1}^{\infty} g^{i}(I)$. The interval $J$ cannot be included in $W$ and so $W \neq \mathbb{S}^{1}$. The number of connected components of $W$ is finite because each one has both endpoints in $\cup_{k=1}^{n} P_{k}$. Let $W_{1}, \ldots, W_{m}$ be the set of all connected components of $W$. We note that for each $i=1,2, \ldots, m$ there exists $j \in\{1,2, \ldots, m\}$ such that $g\left(W_{i}\right) \subset W_{j}$. The set $W$ must contain, at least, one of the periodic orbits, say $P_{i}$, so we can assume that $g^{p}\left(W_{1}\right) \cap W_{1} \neq \emptyset$ where $p$ is the period of $P_{i}$. Then $g^{p}\left(W_{1}\right) \subset W_{1}$. On the other hand, the set $W_{1}$ cannot contain any other point of $P_{i}$. Otherwise, this would imply that $P_{i}$ is reducible which is a contradiction because $g$ coincides with $f$ on $P_{i}$. So, $m$ must be larger than one and $W_{1}$ must contain a point of an orbit $P_{j}$, with $j \neq i$. Let $V$ be a connected component of $\pi^{-1}\left(W_{1}\right)$. Clearly, its diameter is smaller than one. So, if $\frac{r}{p}$ is the rotation number of $P_{i}$, we have that $G^{p}(V) \subset V+r$. Then, for any $x \in W_{1}, \rho_{G}(x)=\frac{r}{p}$. In particular, $\rho_{G}\left(P_{i}\right)=\rho_{G}\left(P_{j}\right)$. Consequently, $\rho_{F}\left(P_{i}\right)=\rho_{F}\left(P_{j}\right)$ contradicting our hypothesis.

For the proofs of Lemma 3.4 and Corollary 3.5 follow from Propositions 3.7.11 and 3.7 .19 of $[1]$.

Lemma 3.4. Let $F$ be a lifting of a continuous circle map of degree one. If $F$ is non-decreasing then

$$
\lim _{n \rightarrow \infty} \frac{F^{n}(y)-y}{n}
$$

exist and is independent on $y \in \mathbb{R}$.

Corollary 3.5. Let $F, G$ and $H$ be liftings of degree one circle maps such that $F$ and $H$ are non-decreasing and $F \leq G \leq H$. If $L_{G}=[\alpha, \beta]$ and $x \in \mathbb{S}^{1}$ then $\rho_{F}(x) \leq \alpha$ and $\rho_{H}(x) \geq \beta$.

Lemma 3.6. Suppose that $F$ is the lifting of a degree one circle map and that $[\alpha, \beta] \subset$ $L_{F}$ where $\alpha<\beta$. Then there exist a map $g \in \mathcal{C}_{1}\left(\mathbb{S}^{1}\right)$ with a lifting $G$ such that

(1) $L_{G}=[\alpha, \beta]$

(2) If for some $x \in \mathbb{R}, G(x) \neq F(x)$ then there exist a neighborhood $U$ of $x$ such that $\left.G\right|_{U}$ is constant.

Proof. From [4] (see also [1, Proposition 3.7.17]) there exist two non-decreasing liftings of degree one circle maps, maps $F_{\alpha}$ and $F_{\beta}$, such that:

(i) $F_{\alpha} \leq F_{\beta}$

(ii) $L_{F_{\alpha}}=\{\alpha\}$

(iii) $L_{F_{\beta}}=\{\beta\}$

(iv) If for some $x \in \mathbb{R}, F_{\alpha}(x) \neq F(x)$ then there exist a neighborhood $U$ of $x$ such that $\left.F_{\alpha}\right|_{U}$ is constant. 
(v) If for some $x \in \mathbb{R}, F_{\beta}(x) \neq F(x)$ then there exist a neighborhood $U$ of $x$ such that $\left.F_{\beta}\right|_{U}$ is constant.

Define a map $G: \mathbb{R} \rightarrow \mathbb{R}$ in the following way:

$$
G(x)= \begin{cases}F_{\alpha}(x) & \text { if } F(x) \leq F_{\alpha}(x) \\ F(x) & \text { if } F_{\alpha}(x) \leq F(x) \leq F_{\beta}(x) \\ F_{\beta}(x) & \text { if } F_{\beta}(x) \leq F(x)\end{cases}
$$

This map is continuous and verifies that $G(x+1)=G(x)+1$. So it is the lifting of a map $g \in \mathcal{C}_{1}\left(\mathbb{S}^{1}\right)$. Clearly the map $G$ satisfies statement (2). Next we will show that $L_{G}=[\alpha, \beta]$. By Corollary 3.5 and the inequalities $F_{\alpha} \leq G \leq F_{\beta}$ we deduce that $L_{G} \subset[\alpha, \beta]$. To prove the other inclusion we note that, from [4], there exists $x_{\alpha} \in \mathbb{S}^{1}$ such that $F_{\alpha}^{n}\left(y_{\alpha}\right)=F^{n}\left(y_{\alpha}\right)$ for each $n \geq 0$ and $y_{\alpha} \in \pi^{-1}\left(x_{\alpha}\right)$. Therefore, from the definition of $G$ it follows that $F_{\alpha}^{n}\left(y_{\alpha}\right)=G^{n}\left(y_{\alpha}\right)$ for each $n \geq 0$ and $y_{\alpha} \in \pi^{-1}\left(x_{\alpha}\right)$. So, from (ii) it follows that $\alpha \in L_{G}$. The fact that $\beta \in L_{G}$ can be shown in a similar way.

Proof of Theorem B. Suppose first that $\alpha=\beta \in L_{F}$. By [4] there exists a point $x \in S^{1}$ such that its orbit has rotation number $\alpha$ and it is twist. Consider $W=\operatorname{Cl}\left(\operatorname{Orb}_{f}(x)\right)$ and observe that $\left.F\right|_{W}$ is still non-decreasing. Define a continuous map $G: \mathbb{R} \rightarrow \mathbb{R}$ in the following way: $\left.F\right|_{W}=\left.G\right|_{W}$ and $G$ is affine on each connected component of $\mathbb{R} \backslash W$. This map $G$ is the lifting of a degree one circle map and it is non-decreasing. Hence, by Lemma 3.4, $L_{G}=\{\alpha\}$. Since $F^{n}(y)=G^{n}(y)$ for every $y \in \pi^{-1}\left(\operatorname{Orb}_{f}(x)\right)$, then $L_{F}(x)=L_{G}(x)=\{\alpha\}=L_{G}=R_{G}(x)=R_{F}(x)$.

Now we consider the case $\alpha<\beta$. Take a sequence of rational numbers $\left\{\frac{p_{i}}{q_{i}}\right\}_{i \in \mathbb{N}} \subset$ $[\alpha, \beta]$ such that $p_{i}$ and $q_{i}$ are coprime and $\frac{p_{2 i}}{q_{2 i}} \searrow \alpha$ and $\frac{p_{2 i+1}}{q_{2 i+1}} \nearrow \beta$ when $i \rightarrow \infty$. Let $g \in \mathcal{C}_{1}\left(\mathbb{S}^{1}\right)$ and $G$ be the maps from Lemma 3.6. From [4] it follows that for each $i$ there exist a twist periodic orbit $P_{i}$ of $g$ with rotation number $\left\{\frac{p_{i}}{q_{i}}\right\}$ such that, if $U$ is a neighborhood of a point $x \in \pi^{-1}\left(P_{i}\right)$, then $\left.G\right|_{U}$ is not constant. We note that, in view of Lemma 3.6(2), we have that $\left.F\right|_{\pi^{-1}\left(P_{i}\right)}=\left.G\right|_{\pi^{-1}\left(P_{i}\right)}$. Without loss of generality we can assume that $\pi(0) \in P_{1}$.

For each $i \in \mathbb{N}$ denote by $X_{i}$ the set $\cup_{j=1}^{i} P_{j}$ and let $I_{1, i}, \ldots, I_{m_{i}, i}$ be the $X_{i}$-basic intervals. Let $\underline{\mathbf{R}}_{i}$ be the code of a point from $P_{i}$ in the rotational $X_{i}$-graph of $g$ and denote its first symbol by $I_{j_{i}, i}$. Since the partition given by $X_{i+1}$ is finer than the one given by $X_{i}$ for each $i$, there exists an $X_{i}$-basic interval containing $I_{j_{i+1}, i+1}$. Denote this interval by $I_{t_{i}, i}$. By Lemma 3.3 there exist an admissible sequence $\underline{\mathbf{S}}_{i}$ for the rotational $X_{i}$-graph of $g$ such that the sequence $\underline{\mathbf{R}}_{i} \underline{\mathbf{S}}_{i} I_{t_{i}, i}^{m_{i}}$ is admissible for some $m_{i} \in \mathbb{Z}$. The sequence $\underline{\mathbf{S}}_{i}$ will be used to "glue" sequences of the form $\underline{\mathbf{R}}_{i}^{n_{i}}$ with $n_{i}$ growing in a "convenient" way with $i$. Let us denote the last symbol of $\underline{\mathbf{S}}_{i}$ by $I_{l_{i}, i}^{h_{i}}$.

Claim 1. There exists a sequence of natural numbers $\left\{n_{i}\right\}_{i \in \mathbb{N}}$ and a sequence of positive reals $\left\{\varepsilon_{i}\right\}_{i \in \mathbb{N}}$ converging to zero when $i$ tends to $\infty$ such that:

(a) If $i$ is odd, then $\frac{p_{i}}{q_{i}}-\varepsilon_{i}<\rho\left(\underline{\mathbf{R}}_{1}^{n_{1}} \underline{\mathbf{S}}_{1} \ldots \underline{\mathbf{R}}_{i}^{n_{i}} \underline{\mathbf{S}}_{i}\right)<\beta$, 
(b) If $i$ is even, then $\alpha<\rho\left(\underline{\mathbf{R}}_{1}^{n_{1}} \underline{\mathbf{S}}_{1} \ldots \underline{\mathbf{R}}_{i}^{n_{i}} \underline{\mathbf{S}}_{i}\right)<\frac{p_{i}}{q_{i}}+\varepsilon_{i}$.

Proof of Claim 1. First take any sequence of positive reals $\left\{\varepsilon_{i}\right\}_{i \in \mathbb{N}}$ tending to zero. Now we will prove (a) and (b) by induction. Since the proofs for $i=1$ and the inductive steps when $i$ is even and when is odd are similar, we are only going to make the proof for the case $i$ is odd.

Suppose that $i$ is odd and we have $n_{1}, \ldots, n_{i}$ verifying conditions (a) and (b). If $\rho\left(\underline{\mathbf{R}}_{1}^{n_{1}} \underline{\mathbf{S}}_{1} \ldots \underline{\mathbf{R}}_{i}^{n_{i}} \underline{\mathbf{S}}_{i} \underline{\mathbf{S}}_{i+1}\right)=\frac{a}{b}$ then $\rho\left(\underline{\mathbf{R}}_{1}^{n_{1}} \underline{\mathbf{S}}_{1} \ldots \underline{\mathbf{R}}_{i}^{n_{i+1}} \underline{\mathbf{S}}_{i} \underline{\mathbf{R}}_{i+1}^{n} \underline{\mathbf{S}}_{i+1}\right)=\frac{a+n p_{i+1}}{b+n q_{i+1}}$ which converges to $\frac{p_{i+1}}{q_{i+1}}$ as $n$ tends to infinity. So, choosing $n_{i+1}$ large enough, (b) is satisfied. This ends the proof of Claim 1.

Claim 2. For each $i \in \mathbb{N}$ let $\rho\left(\underline{\mathbf{R}}_{i}^{n_{i}} \underline{\mathbf{S}}_{i}\right)=\frac{a_{i}}{b_{i}}$ where $b_{i}=\operatorname{len}\left(\underline{\mathbf{R}}_{i}^{n_{i}} \underline{\mathbf{S}}_{i}\right)$. Then there exist a non increasing sequence of closed intervals $\left\{I_{i}\right\}_{i \in \mathbb{N}}$ of $\mathbb{S}^{1}$ such that:

(i) If $x \in I_{i}$ then $x$ f-follows $\underline{\mathbf{R}}_{1}^{n_{1}} \underline{\mathbf{S}}_{1} \ldots \underline{\mathbf{R}}_{i}^{n_{i}} \underline{\mathbf{S}}_{i}$

(ii) $G^{k_{i}}\left(\widetilde{I}_{i}\right)=\widetilde{I}_{l_{i}, i}+\left(\sum_{j=1}^{i} a_{j}\right)-h_{i}$ where $k_{i}=\left(\sum_{j=1}^{i} b_{j}\right)-1$.

Proof of Claim 2. The case $i=1$ follows from Lemma 3.2. Suppose now that there exist intervals $I_{1}, \ldots, I_{i}$ verifying (i) and (ii). Since the sequence $\underline{\mathbf{S}}_{i} I_{t_{i}, i}^{m_{i}}$ is admissible, from (ii), it follows that $\widetilde{I}_{j_{i+1}, i+1}+\sum_{j=1}^{i} a_{j} \subset \widetilde{I}_{t_{i}, i}+\sum_{j=1}^{i} a_{j} \subset G^{k_{i}+1}\left(\widetilde{I}_{i}\right)$. On the other hand, by Lemma 3.2 there exist an interval $J \subset I_{j_{i+1}, i+1}$ such that every $x \in J$ $f$-follows the sequence $\underline{\mathbf{R}}_{i+1}^{n_{i+1}} \underline{\mathbf{S}}_{i+1}$ and $G^{b_{i+1}-1}(\widetilde{J})=\widetilde{I}_{l_{i+1}, i+1}+a_{i+1}-h_{i+1}$. Taking $I_{i+1}$ as the subinterval of $I_{i}$ such that $G^{k_{i}+1}\left(\widetilde{I}_{i+1}\right)=\widetilde{J}+\sum_{j=1}^{i} a_{j}$, Claim 2 follows.

Now take $x$ in the intersection of all intervals $I_{i}$ from Claim 2. By Claim 1 and Lemma 3.1 we have that $[\alpha, \beta] \subset L_{G}(x)$. On the other hand, $L_{G}(x) \subset R_{G}(x) \subset L_{G}=$ $[\alpha, \beta]$. So, $L_{G}(x)=R_{G}(x)=[\alpha, \beta]$. Observe that because of the definition of $G$ (see the proof of Lemma 3.6), for any finite set $P \subset \mathbb{S}^{1}$, the rotational $P$-graph of $g$ is a subgraph

of the rotational $P$-graph of $f$. Therefore, Claims 1 and 2 also hold for $F$ instead of $G$. Let $\left\{I_{i}^{F}\right\}_{i \in \mathbb{N}}$ be the sequence given by Claim 2 for $F$ and take $y \in \cap_{i=1}^{\infty} I_{i}^{F}$. By the definition of $x$ and $y$ and Lemma 3.1 we get that $L_{G}(x)=R_{G}(x)=L_{F}(y)=R_{F}(y)$. This ends the proof of the theorem.

\section{Example}

In this section we present an example of a map $f \in \mathcal{C}_{1}\left(\mathbb{S}^{1}\right)$ with lifting $F$ such that for some $x \in \mathbb{S}^{1}$ the following statements hold:

(1) $L_{F}(x)=\left\{\rho_{F}(x)\right\}$ is strictly included in $R_{F}(x)$.

(2) $\cup_{z \in \omega_{f}(x)} L_{F}(z)$ is not convex. 
(3) $R_{F}^{e}(x)$ only contains the extremal points of $R_{F}(x)$, therefore its interior is empty but the interior of $R_{F}(x)$ is the set $(0,1)$.

We will say that a point $x \in \mathbb{S}^{1} f$-follows an infinite sequence $A_{1}^{k_{1}} A_{2}^{k_{2}} \ldots$ if it $f$-follows the sequence $A_{1}^{k_{1}} A_{2}^{k_{2}} \ldots A_{n}^{k_{n}}$ for each $n \in \mathbb{N}$.

Example 4.1. Take two disjoint intervals $I_{0}, I_{1} \subset \mathbb{S}^{1}$ and take $P$ as the set of endpoints of $I_{0}$ and $I_{1}$. Consider a map $f \in \mathcal{C}_{1}\left(\mathbb{S}^{1}\right)$ with lifting $F$ such that in its rotational $P$ graph there exist one arrow from $I_{i}$ to $I_{j}$ with label $i$ where $i, j \in\{0,1\}$ and suppose that $F$ is affine on $\widetilde{I}_{0}$ and $\widetilde{I}_{1}$. Take a point $x$ which $f$-follows the sequence $\prod_{i \in \mathbb{N}}\left(I_{0}^{0}\right)^{i}\left(I_{1}^{1}\right)^{i}$. Then by Lemma 3.1, $\rho_{F}(x)=\frac{1}{2}$. Clearly, each element of the $\omega$-limit of $x f$-follows a sequence in the set

$$
\left\{\left(I_{0}^{0}\right)^{i}\left(I_{1}^{1}\right)^{\infty},\left(I_{1}^{1}\right)^{i}\left(I_{0}^{0}\right)^{\infty}: i \in \mathbb{N} \cup\{0\}\right\}
$$

Again by Lemma 3.1, we have that $\cup_{z \in \omega_{f}(x)} L_{F}(z)=\{0,1\}$. By Theorem A and the fact that the interior of $R_{F}$ is $(0,1)$ this shows (1) and (2).

To prove (3) we note that, since $F$ is expansive in $\widetilde{I}_{0}$ and $\widetilde{I}_{1}$, for each infinite sequence of symbols $I_{0}^{0}$ and $I_{1}^{1}$, there exists a unique point $f$-following it. If $\mu \in M_{e}^{f}\left(\mathrm{Cl}\left(\mathrm{Orb}_{f}(x)\right)\right)$ then $\sup (\mu) \subset \operatorname{Rec}\left(\left.f\right|_{\mathrm{Cl}\left(\operatorname{Orb}_{f}(x)\right)}\right) \subset \omega_{f}(x)$. So, $M_{e}^{f}\left(\mathrm{Cl}\left(\operatorname{Orb}_{f}(x)\right)\right)=\left\{\mu_{0}, \mu_{1}\right\}$ where $x_{i}$ is the only point that $f$-follows the sequence $\left(I_{i}^{i}\right)^{\infty}$ and $\mu_{i}=\delta_{x_{i}}$. Then we have

$R_{e}^{F}(\mathrm{Cl}(\mathrm{Orb}(x)))=\{0,1\}$ and $R^{F}(\mathrm{Cl}(\mathrm{Orb}(x)))=R_{m}^{F}(\mathrm{Cl}(\mathrm{Orb}(x)))=[0,1]$. This shows (3).

\section{References}

[1] Ll. Alsedà, J. Llibre and M. Misiurewicz, Combinatorial dynamics and entropy in dimension one, Advanced Series in Nonlinear Dynamics, Vol. 5, World Scientific, Singapore, 1993.

[2] L. Block, J. Guckenheimer, M. Misiurewicz and L. S. Young, Periodic points and topological entropy of one-dimensional maps, Lecture Notes in Math. 819, pp. 18-34, Springer, Berlin, 1980.

[3] R. Bamón, I. Malta, M. J. Pacífico and F. Takens, Rotation intervals of endomorphisms of the circle Ergod. Th. \& Dynam. Sys. 4, (1984), 493-498.

[4] A. Chenciner, J. -M. Gambaudo and C. Tresser, Une remarque sur la structure des endomorphismes de degré 1 du cercle C. R. Acad. Sci. Paris, Sér. I Math. 299 (1984), 145-148.

[5] A. Fathi Nombres de rotation sur le cercle et le tore, Note de lécole d'été sur les systèmes dynamiques en petites dimensions, Grenoble-20 juin-8 jullet, 1994.

[6] R. Ito, Rotation sets are closed, Math. Proc. Camb. Phil. Soc. 89 (1981), 107-111. 
[7] J. Llibre and R. S. MacKay, Rotation vectors and entropy for homeomorphisms of the torus isotopic to the identity Ergod. Th. \& Dynam. Sys. 11 (1991), 115-128.

[8] M. Misiurewicz and K. Ziemian, Rotation sets for maps of tori, J. London Math. Soc. (2) 40 (1989) 490-506.

[9] M. Misiurewicz and K. Ziemian, Rotation sets and ergodic measures for maps of torus homeomorphisms, Fundam. Math. 137 (1991), 45-52.

[10] S. Newhouse, J. Palis and F. Takens, Bifurcations and stability of families of diffeomorphisms, Inst. Hautes Etudes Sci. Publ. Math. 57 (1983), 5-71.

[11] W. Rudin, Functional analysis, McGraw-Hill, New York, 1973.

[12] P. Walters An introduction to ergodic theory Graduate Texts in Math. 79, Springer, Berlin, 1982. 